\title{
Combination Therapy of Angiotherapy, Radiation Therapy, and Radiofre- quency Ablation for Pulmonary Metastasis of Hepatocellular Carcinoma Accompanied by Nontuberculous Mycobacteria
}

\author{
Sang Hyun Park', Seul Ki Kim', Ji Hye Kim', Seokgyo Seo', Hyun Pyo Hong², Soo-Youn Ham², Byung Ik Kim¹ \\ 'Division of Gastroenterology, Department of Internal Medicine, ${ }^{2}$ Department of Radiology, Kangbuk Samsung Hospital, Sungkyunkwan \\ University School of Medicine, Seoul, Korea
}

Received Feb. 12, 2019

Revised Mar. 12, 2019

Accepted Mar. 12, 2019
With the advances in hepatocellular carcinoma (HCC) treatment, the lung metastasis of HCC is becoming increasingly important. In treating the lung metastasis of HCC, a multidisciplinary approach can lead to better results than systemic chemotherapy alone. Here, we report on a patient who presented with pulmonary masses, while the HCC was being controlled in the abdominal cavity. The presence of nontuberculous mycobacteria was identified during the diagnosis of the pulmonary masses. The pulmonary metastases of HCC were treated with a combination of angiotherapy, radiation therapy, and radiofrequency ablation. The patient showed a satisfactory progress with this multidisciplinary localized treatment. We report the clinical progress and review the recent literature regarding the treatment of pulmonary metastasis without intrahepatic HCC herein. (J Liver Cancer 2019;19:79-84)

Keywords: Hepatocellular, carcinoma; Neoplasm metastasis; Therapeutics

\section{INTRODUCTION}

Hepatocellular carcinoma (HCC) is the seventh most common malignant neoplasm in the world, ${ }^{1}$ and it has a high incidence in Korea. ${ }^{2}$ The lungs are the most common site of the extrahepatic metastasis of HCC. In patients who develop pulmonary metastasis without intrahepatic HCC, surgery could result in successful outcomes. ${ }^{3}$ However, most patients with pulmonary metastases may not be operable, because of the location of the lung lesion, surgical technical problems or

\section{Corresponding author : Byung Ik Kim}

Division of Gastroenterology, Department of Internal Medicine, Kangbuk Samsung Hospital, Sungkyunkwan University School of Medicine, 29 Saemunan-ro, Jongno-gu, Seoul 03181, Korea

Tel. +82-2-2001-2055, Fax. +82-2-2001-2049

E-mail; bik.kim@samsung.com

https://orcid.org/0000-0002-7215-7715 poor pulmonary function. In this situation, a multidisciplinary approach can lead to better results. ${ }^{4,5}$ We report a patient with pulmonary metastases without intrahepatic HCC, who was successfully treated with the combination of angiotherapy, radiotherapy, and radiofrequency ablation.

\section{CASE REPORT}

\section{Clinical findings}

A 61-year-old man visited the emergency room with massive hemoptysis and cough. The patient had been diagnosed and treated for tuberculosis in his twenties. The patient was currently taking entecavir for hepatitis B and liver cirrhosis. The patient had been diagnosed with HCC 6 years ago. Initially, the patient had a 10-cm single mass. The stage of HCC was Union for International Cancer Control (UICC) stage II, 
and Barcelona Clinic Liver Cancer (BCLC) stage A. The patient underwent hepatic lobectomy and cholecystectomy. The histologic finding of the resected tissue showed acinar histologic patterns, a classical type of a tumor cell group, Edmondson-Steiner grade II/IV, 50\% tumor necrosis, and no vascular invasion. Two years later, three recurrent nodular HCC lesions were found on follow-up liver dynamic contrast-enhanced computed tomography (CECT). At that time, the patient underwent one course of transarterial chemoembolization (TACE) and one course of radiofrequency ablation (RFA). Since then, he did not exhibit recurrence in the abdominal cavity.

One year ago, a single lung mass was found on follow-up liver dynamic CECT. The patient underwent chest CECT, which revealed a $35-\mathrm{mm}$ left upper lobe (LUL) mass and a $10-\mathrm{mm}$ right lower lobe (RLL) mass (Fig. 1). Initially, we considered performing surgical biopsy. However, as both lesions were located centrally, the patient would require left pneumonectomy for the LUL mass and RLL lobectomy for the RLL mass, which could result in poor quality of life. We also considered percutaneous transthoracic needle biopsy (PCNB). However, PCNB could not be performed, because pulmonary vessels surrounded both lung lesions. On CECT images, several mediastinal lymph node enlargements were also found. Instead of obtaining tissue directly from the lung masses, we planned to infer their pathology indirectly by determining the pathology of the mediastinal lymph nodes. The patient underwent video-assisted thoracoscopic surgery to obtain subcarinal lymph node tissue. The histologic finding confirmed that the tissue had granulomatous inflammation with necrosis and tested positive for nontuberculous mycobacteria (NTM) by polymerase chain reaction. Based on this result, antibiotics for the NTM infection were prescribed for one year. The size of the lung masses did not decrease after the treatment. After concluding that there was no therapeutic effect, the patient discontinued taking antibiotics for the NTM infection.

After five months, the patient revisited the emergency room with massive hemoptysis and cough. In the emergency room, his blood pressure was 130/70 $\mathrm{mmHg}$, body temperature was $36.5^{\circ} \mathrm{C}$, pulse rate was 102 beats/min, and respiration rate was 25 breaths/min. There was no cardiac murmur. There was a coarse crackle in the right lung field. There were no other unusual findings on abdominal, skin, and joints examinations.

In the complete blood count test, the white blood cell

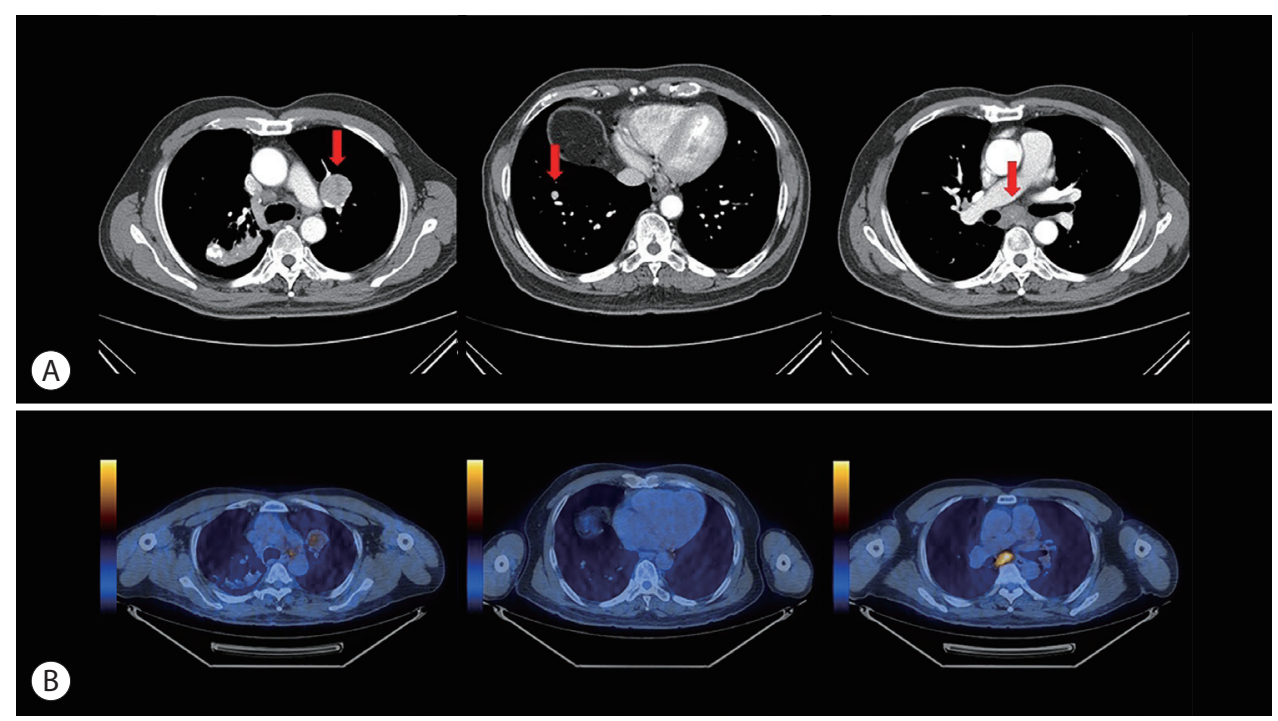

Figure 1. CECT scan revealing well-defined, round masses $(35 \mathrm{~mm}$ in the LUL and $10 \mathrm{~mm}$ in the RLL, each is marked with an arrow in the left and middle images) and enlarged subcarinal lymph node (arrow in the right image) with faint central calcification. (B) PET-CT performed one week after CECT showing increased FDG uptakes in the LUL mass and subcarinal lymph node. CECT, contrastenhanced computed tomography; LUL, left upper lobe; RLL, right lower lobe; PET-CT, positron emission tomography-computed tomography; FDG, fluorodeoxyglucose. 
count was $5,920 / \mathrm{mm}^{3}$ (neutrophils, $58.3 \%$; eosinophils, 2.4\%; basophils, 0.8\%; lymphocytes, 32.6\%; and monocytes, $5.9 \%$ ), hemoglobin level was $13.4 \mathrm{~g} / \mathrm{dL}$, and platelet count was $194,000 / \mathrm{mm}^{3}$. Aspartate aminotransferase and alanine aminotransferase levels were 25 and 23 IU/L, respectively. The serum albumin level was $4.7 \mathrm{~g} / \mathrm{dL}$. The prothrombin time was 10.8 seconds (normal range, 9.5-12.1) and activated partial thromboplastin time was 29.0 seconds (normal range, 26.4-36.7). The alpha-fetoprotein level was in the normal range at $1.93 \mathrm{ng} / \mathrm{mL}$. The level of the proteins induced by vitamin $\mathrm{K}$ absence or antagonist-II (PIVKA-II) increased to $1,044 \mathrm{mAU} / \mathrm{mL}$.

\section{Image findings}

The CECT performed in the emergency room showed that there was no active bleeding, but ground-glass opacity was found, which was caused by the aspirated blood. The previously identified lung masses had increased in size. The LUL mass increased from $3.9 \mathrm{~cm}$ to $4.4 \mathrm{~cm}$, and the RLL mass increased from $1.1 \mathrm{~cm}$ to $1.6 \mathrm{~cm}$ (Fig. 2).

\section{Diagnosis and treatment progress}

It was estimated that the massive hemoptysis had been caused by dilated vessels surrounding the lung masses. To plan the treatment of the lung masses, we concluded that

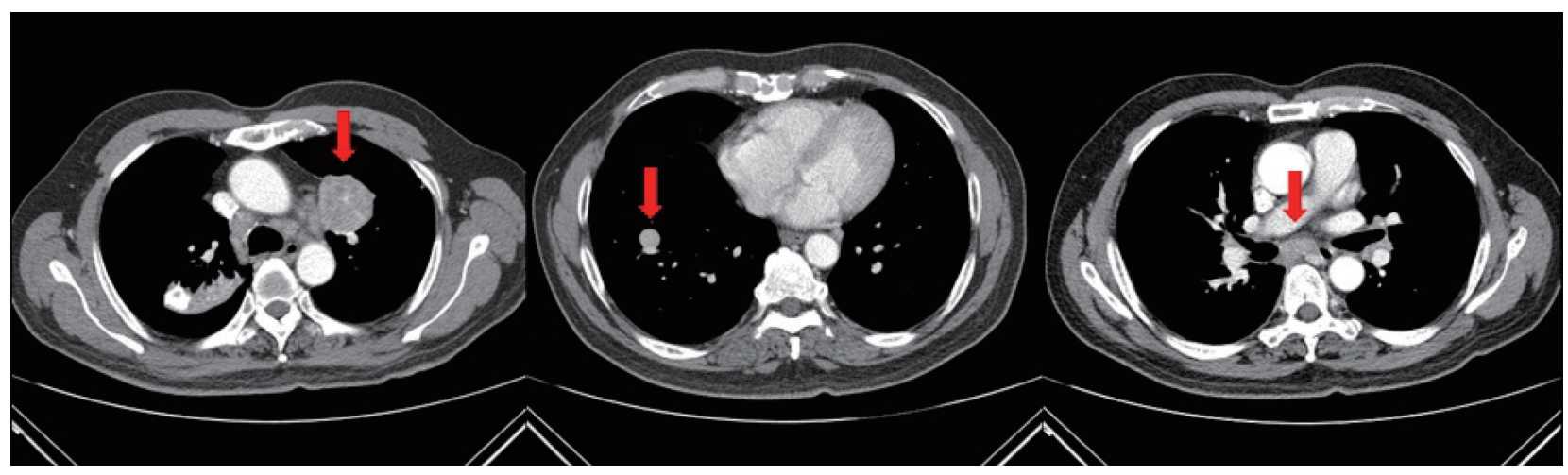

Figure 2. Follow-up CECT showing interval enlarged LUL mass (arrow in the left image) with heterogeneous contrast enhancement and internal lower densities, as well as an interval enlarged RLL mass (arrow in the middle image). Both lung masses appear suggestive of pulmonary metastasis. When compared with the initial CECT scan, interval increases in the sizes of mediastinal lymph nodes (arrow in the right image) can be seen. CECT, contrast-enhanced computed tomography; LUL, left upper lobe; RLL, right lower lobe.
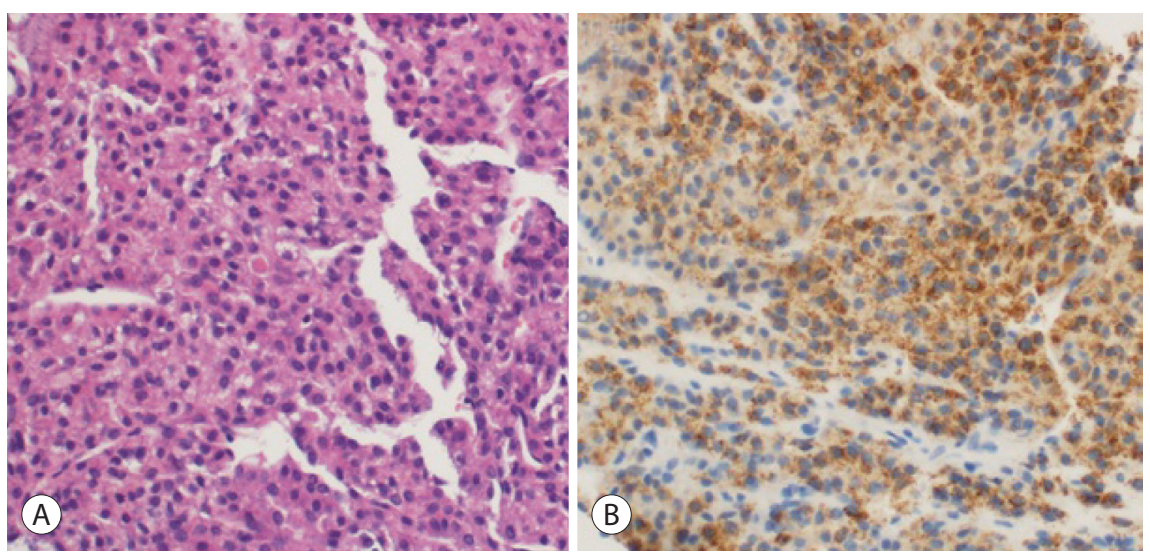

Figure 3. Histopathology of the pulmonary metastasis. (A) H\&E staining of the lung mass showing metastatic hepatocellular carcinoma (original magnification, $\times 200$ ). (B) Tumor cells appear positive for hepatocyte-specific antigen (original magnification, $\times 200$ ). H\&E, hematoxylin and eosin. 


\section{Journal of Liver Cancer}
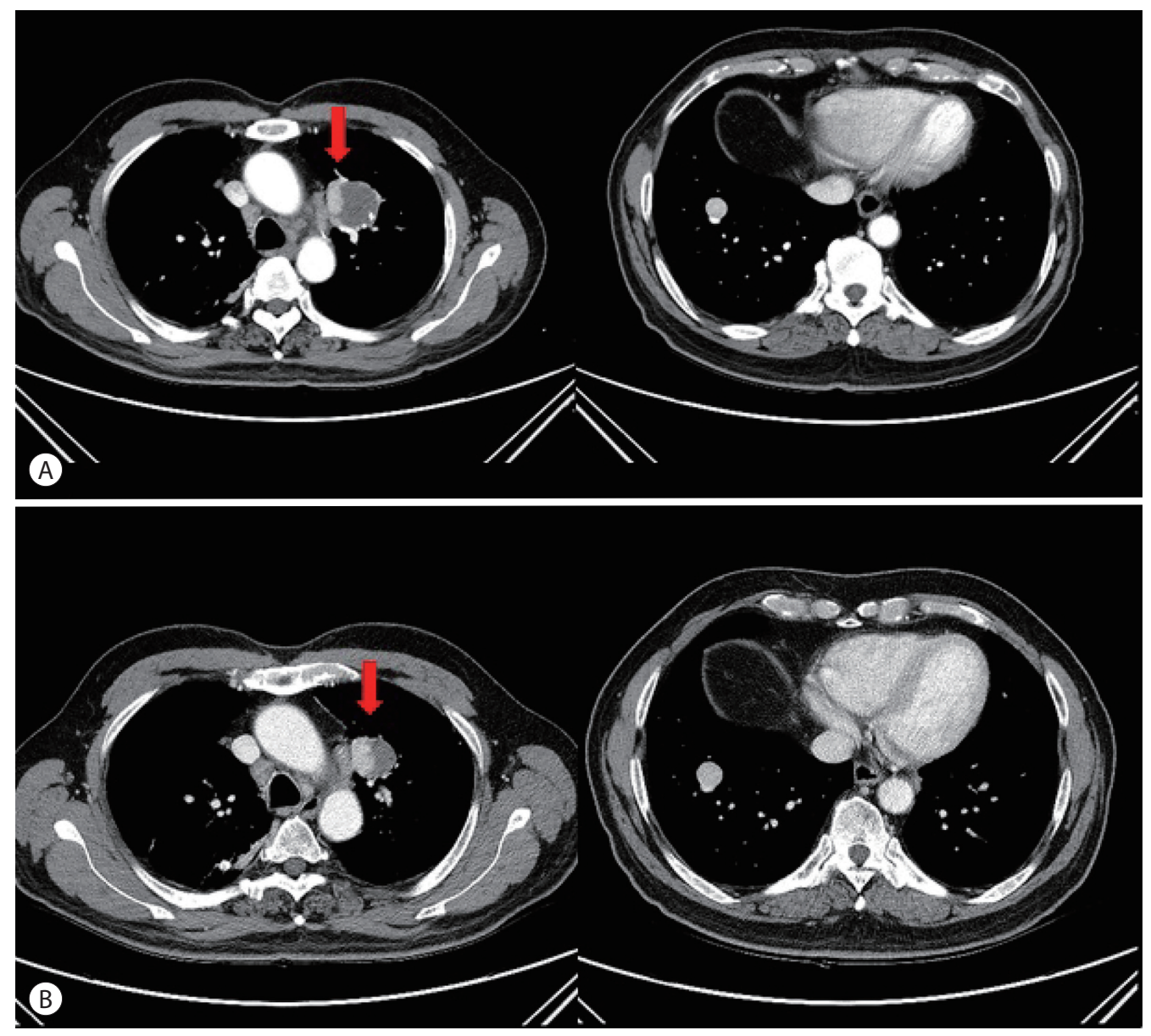

Figure 4. (A) CECT performed one month after BAE showing that the size of the LUL mass (arrow) decreased from $44 \mathrm{~mm}$ to $40 \mathrm{~mm}$. However, there an enhancing portion remained, suggesting viable foci. (B) CECT performed one month after radiotherapy showing that, in the LUL mass (arrow), the enhancing lesion elongated at the medial aspect. The size of the RLL mass increased from $15 \mathrm{~mm}$ to $17 \mathrm{~mm}$. CECT, contrast-enhanced computed tomography; BAE, bronchial artery embolization; LUL, left upper lobe; RLL, right lower lobe.
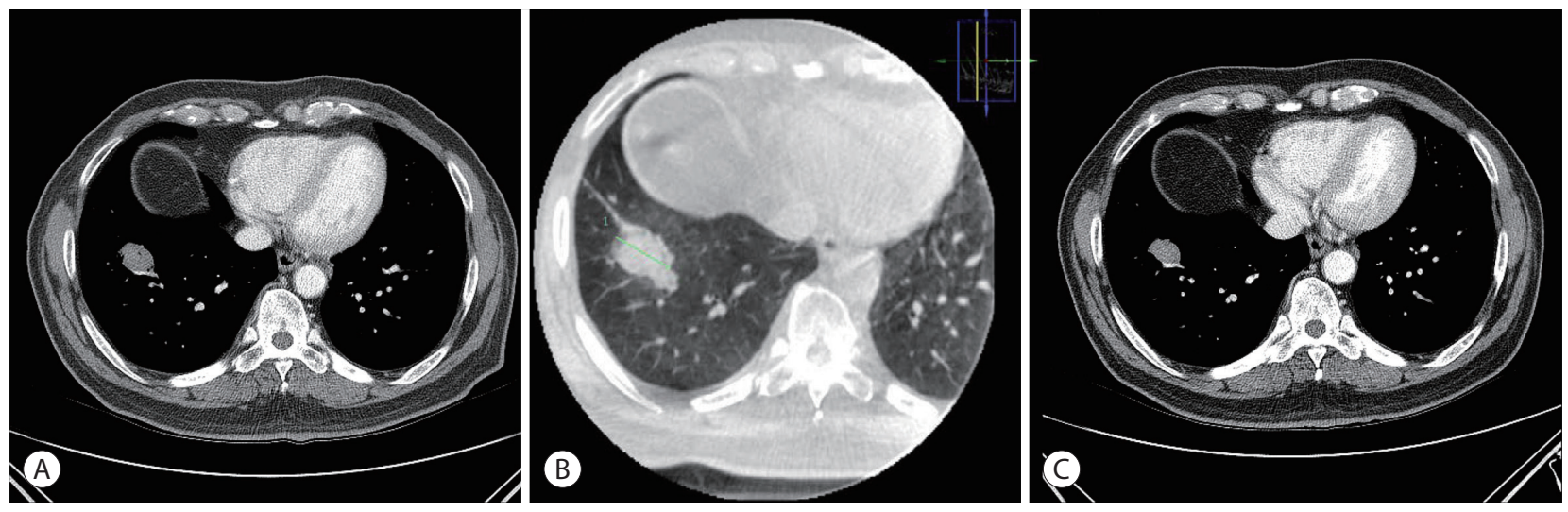

Figure 5. (A) The left CECT scan was performed one month after RFA. Immediate follow-up CECT revealing an increase in the size of the RLL nodule. (B) Cone-beam CT was performed during the RFA procedure. Ground-glass opacity formed around the RLL mass. (C) The right CECT scan was performed three months after RFA. The size of the RLL mass decreased, and there was no viable portion in the mass. CECT, contrast-enhanced computed tomography; RFA, radiofrequency ablation; $\mathrm{RLL}$, right lower lobe; $\mathrm{CT}$, computed tomography. 
tissue biopsy was necessary. On consultation with the interventional radiologist, we planned to conduct bronchial artery embolization (BAE) and PCNB consecutively. The BAE was first performed to treat the massive hemoptysis, followed by the PCNB for the LUL mass biopsy. The patient did not exhibit hemoptysis after the procedure. The histologic finding of the obtained tissue confirmed HCC (Fig. 3). Finally, the patient was diagnosed with pulmonary metastasis without a remaining HCC lesion in the abdominal cavity (UICC stage IVB and BCLC stage C). The disease was classified according to the Child-Turcotte-Pugh classification as A (score 5).

On follow-up CECT, the size of the LUL mass was reduced, and the size of the RLL mass remained the same (Fig. $4 \mathrm{~A})$. The patient received radiotherapy for the LUL mass. The total cumulative dose of 3,500 cGy radiation was administered in seven fractions for the LUL mass. On follow-up CECT, there was no radiologic change in the LUL mass, but the size of the RLL mass increased (Fig. 4B). The patient underwent RFA for the RLL mass.

Currently, there is no viable portion of the RLL mass on follow-up CECT (Fig. 5). The level of the tumor marker PIVKA-II reduced to $27 \mathrm{mAU} / \mathrm{mL}$, which is within its normal range.

\section{DISCUSSION}

The lungs are the most frequent site for the HCC metastasis. Sorafenib is usually chosen as the treatment for an intraabdominal HCC lesions with pulmonary metastasis. However, few patients achieve a successful response with molecular targeted therapy (MTT), because of tumor heterogeneity and complex cell signal transduction. ${ }^{6}$ In cases of isolated pulmonary metastasis, without an intraabdominal HCC lesion, an active multidisciplinary approach can produce good results.

This case is meaningful in suggesting two lessons. First, physicians should keep in mind that a pulmonary lesion and lymph node enlargement could have different pathologies. Second, a combination of localized therapies should be considered for the treatment of isolated pulmonary metastases.

In this case, the presence of NTM was confirmed in the lymph nodes, and HCC was confirmed in the pulmonary nodules. There was histologic discrepancy between the pulmonary lesion and the mediastinal lymph node. Independently of the pulmonary lesion, mediastinal lymph node enlargement can also be observed in double primary cancer or benign diseases, such as tuberculosis, NTM infection, and sarcoidosis. ${ }^{7}$ The prevalence of NTM infection is increasing in Korea. ${ }^{8}$ In this context, the possibility of differences between pulmonary lesion and mediastinal lymph node pathologies should be considered.

The standard treatment of choice for isolated pulmonary metastases of HCC without intraabdominal lesions is surgical resection. ${ }^{3}$ However, surgery should be performed in selected patients, because only few patients could experience benefits, such as prolonged survival time following surgery. Indications for surgical resection include controlled intraabdominal HCC, no metastases to organs other than the lungs, complete resection of metastatic lesions, and sufficient pulmonary function for surgery. ${ }^{9}$ As the proportion of patients meeting these conditions is small, most patients cannot undergo surgical resection.

In such cases, MTT could be considered. Sorafenib and regorafenib have proven effectiveness in patients with advanced HCC. However, a multidisciplinary approach including hepatologists, surgeons, interventional radiologists, and oncologists is preferred because of the heterogeneity in presentation of HCC among patients. ${ }^{4,10}$ Physicians should consider to administer multimodality treatments involving liver transplantation, hepatic resection, RFA, transarterial embolization (TAE), radiotherapy, and systemic chemotherapy.

In this case, BAE was conducted prior to PCNB to treat and prevent hemoptysis. BAE has been demonstrated to be an effective and safe treatment for malignant hemoptysis. ${ }^{11} \mathrm{BAE}$ also had a therapeutic effect on the lung masses. A reduction of the LUL mass size was noted after BAE on follow-up CECT. Embolization therapies play an increasingly important role in patients with advanced HCC with extrahepatic spread. ${ }^{5,12}$ Among embolization therapies, TACE is probably the most widely practiced embolization therapy for HCC. Occasionally, TAE is used for HCC instead of TACE. The goal of TAE is to induce ischemic necrosis by selective catheter-di- 
rected delivery of vaso-occlusive materials directly into the arteries supplying the tumor. ${ }^{12}$

On follow-up CECT, the contrast medium accumulated in the medial side of the LUL mass, which required further treatment. RFA and radiation therapy could both be considered for localized treatment. ${ }^{13}$ Radiation therapy was chosen based on the location of the metastatic lesion, because the lesion size was large and the location of the mass was difficult to reach with RFA.

RFA is a treatment mainly performed as a localized treatment for intraabdominal HCC, including adrenal metastasis. Recently, RFA has also been performed as a localized treatment for pulmonary metastasis of HCC. ${ }^{13,14}$ RFA can be used in cases wherein the size of the mass is small and the mass does not invade mediastinal structures. In this case, the RLL mass was suitable for RFA.

Currently, the patient status is stable. Further treatment will be considered for the LUL mass if either the uptake in positron emission tomography-computed tomography or PIVKA II level increases. This case is clinically relevant because three localized therapies were applied (radiotherapy, RFA, and angiotherapy) and the patient achieved satisfactory outcomes.

\section{Conflicts of Interest}

The authors have no conflicts to disclose.

\section{REFERENCES}

1. Bray F, Ferlay J, Soerjomataram I, Siegel RL, Torre LA, Jemal A. Global cancer statistics 2018: GLOBOCAN estimates of incidence and mortality worldwide for 36 cancers in 185 countries. CA Cancer J Clin 2018;68:394-424.
2. Kim BH, Park JW. Epidemiology of liver cancer in South Korea. Clin Mol Hepatol 2018;24:1-9.

3. Chan KM, Yu MC, Wu TJ, Lee CF, Chen TC, Lee WC, et al. Efficacy of surgical resection in management of isolated extrahepatic metastases of hepatocellular carcinoma. World J Gastroenterol 2009;15:5481-5488.

4. Aramaki M, Kawano K, Kai T, Yokoyama H, Morii Y, Sasaki A, et al. Treatment for extrahepatic metastasis of hepatocellular carcinoma following successful hepatic resection. Hepatogastroenterology 1999:46:2931-2934.

5. Hiraki T, Gobara H, Takemoto M, Mimura H, Mukai T, Himei K, et al. Percutaneous radiofrequency ablation combined with previous bronchial arterial chemoembolization and followed by radiation therapy for pulmonary metastasis from hepatocellular carcinoma. J Vasc Interv Radiol 2006;17:1189-1193.

6. Llovet JM, Villanueva A, Lachenmayer A, Finn RS. Advances in targeted therapies for hepatocellular carcinoma in the genomic era. Nat Rev Clin Oncol 2015;12:408-424.

7. Lee SJ, Lim SY, Yoo TK, Kim SK, Kim YG, Lee HJ, et al. A case of synchronous lung squamous cell carcinoma and diffuse large B-cell Iymphoma. Korean J Med 2018;93:300-305.

8. Kee SJ, Suh SP. Increasing burden of nontuberculous mycobacteria in Korea. J Korean Med Sci 2017;32:1215-1216.

9. Petrella F, Diotti C, Rimessi A, Spaggiari L. Pulmonary metastasectomy: an overview. J Thorac Dis 2017;9(Suppl 12):S1291-S1298.

10. Guy J, Kelley RK, Roberts J, Kerlan R, Yao F, Terrault N. Multidisciplinary management of hepatocellular carcinoma. Clin Gastroenterol Hepatol 2012;10:354-362.

11. Mehta AS, Ahmed O, Jilani D, Zangan S, Lorenz J, Funaki B, et al. Bronchial artery embolization for malignant hemoptysis: a single institutional experience. J Thorac Dis 2015;7:1406-1413.

12. Kishore S, Friedman T, Madoff DC. Update on embolization therapies for hepatocellular carcinoma. Curr Oncol Rep 2017;19:40.

13. Ryoo HM. Management of pulmonary metastasis. Korean J Med 2011;81:289-299.

14. Hachisuka Y, Uomoto M, Fujisawa T, Mogami H. First reported case of lung metastasis from hepatocellular carcinoma successfully treated by ultrasound-guided radiofrequency ablation using ultrasonic contrast agent. Gan To Kagaku Ryoho 2009;36:2657-2659. 